\title{
Blackbody-radiation-induced facilitated excitation of Rydberg atoms in optical tweezers
}

\author{
Lorenzo Festa $\odot,{ }^{1,2}$ Nikolaus Lorenz $\odot,{ }^{1,2}$ Lea-Marina Steinert, ${ }^{1,2,3}$ Zaijun Chen, ${ }^{1,2}$ Philip Osterholz, ${ }^{1,2,3}$ \\ Robin Eberhard, ${ }^{1,2,3}$ and Christian Gross $\oplus^{1,2,3, *}$ \\ ${ }^{1}$ Max-Planck-Institut für Quantenoptik, 85748 Garching, Germany \\ ${ }^{2}$ Munich Center for Quantum Science and Technology (MCQST), 80799 München, Germany \\ ${ }^{3}$ Physikalisches Institut, Eberhard Karls Universität Tübingen, 72076 Tübingen, Germany
}

(Received 26 March 2021; accepted 3 January 2022; published 19 January 2022)

\begin{abstract}
Blackbody radiation, omnipresent at room temperature, couples nearby Rydberg states. The resulting state mixture features strong dipolar interactions, which may induce dephasing in a Rydberg many-body system. Here we report on a single atom resolved study of this state contamination and the emerging pairwise interactions in optical tweezers. For near-resonant laser detuning we observe characteristic correlations with a length scale set by the dipolar interaction. Our study reveals the microscopic origin of avalanche excitation observed in previous experiments.
\end{abstract}

DOI: 10.1103/PhysRevA.105.013109

Tailored many-body systems can be engineered from atomic ensembles laser-coupled to Rydberg states. In combination with optical tweezers this forms a versatile platform for quantum simulation and computation [1-7]. Understanding decoherence channels is of prime importance for these emerging applications of Rydberg atoms. At room temperature, blackbody radiation is known to incoherently drive transitions between nearby Rydberg states, a process often dominating the decay rate of a Rydberg state [8]. In a many-body setting, the resulting state contamination with Rydberg states of opposite parity opens extremely strong dipolar interaction channels. These uncontrolled interactions lead to dephasing, which may severely limit the coherence time for ensembles of Rydberg atoms. Previous works have observed and studied the presence of interaction induced dephasing and line broadening spectroscopically in a bulk setting [9-14]. A scaling analysis [9] and dynamic experiments [13] pointed towards blackbody radiation induced state contamination to trigger an avalanche excitation process. Dipolar interactions cause level shifts, such that the normally off-resonant laser becomes resonant. This facilitated excitation results in quick population build up in the Rydberg state. Due to the high probability to undergo a blackbody-radiation-induced state change, even more contaminant atoms are produced speeding up the facilitation process. Mean-field models have been employed to explain this effect, but they have shown large quantitative deviation from the data. This triggered a refined theoretical

\footnotetext{
*christian.gross@uni-tuebingen.de
}

Published by the American Physical Society under the terms of the Creative Commons Attribution 4.0 International license. Further distribution of this work must maintain attribution to the author(s) and the published article's title, journal citation, and DOI. Open access publication funded by the Max Planck Society. analysis pointing out the importance of correlations between the excited Rydberg atoms [15].

Here we report on a study of the state contamination induced interactions with neutral atoms individually trapped in a two-dimensional optical tweezer array. Similar to prior experiments in bulk, we near-resonantly laser couple the atoms to a Rydberg state [9-14]. This realizes the setting of Rydberg dressing [16-20], a versatile strategy to realize complex Hamiltonians for the study of quantum magnets [21-23] and to prepare resource states for quantum metrology [17,24-26]. Coherent evolution under Rydberg-induced interactions has been reported in small systems [27-30] or for relatively short times [31] and avalanche excitation has been observed as one limiting process [28,29]. The single atom resolved tweezer system allows us to probe the excited Rydberg atoms one-byone and to study the facilitation process in the pairwise limit outside of the avalanche regime. We extract the characteristic correlation length and directly show that this matches the length scale set by the dipolar interactions.

In our experiment we use ${ }^{39} \mathrm{~K}$ atoms initially prepared in the $|g\rangle=\left|4 S_{1 / 2}, F=2, m_{F}=2\right\rangle$ ground state. We laser couple the atoms to the $\left|r_{0}\right\rangle=\left|62 P_{1 / 2}, m_{J}=-1 / 2\right\rangle$ Rydberg state by an ultraviolet laser at $286 \mathrm{~nm}$ with wave vector $\mathbf{k}_{U V}$, Rabi frequency $\Omega$ and detuning $\Delta$ (see Fig. 1). The circular polarized Rydberg laser beam with a waist of $20 \mu \mathrm{m}$ is propagating in the $x$ direction, parallel to the magnetic field of $10 \mathrm{G}$. The ground state atoms are individually trapped in holographically generated two-dimensional optical tweezer arrangements using laser light at $1064 \mathrm{~nm}$. In each experimental run about half of the tweezers are randomly loaded with a single atom and the occupation of the traps is detected using fluorescence imaging. A typical image is shown in Fig. 1(c) for a $3 \times 16$ array. In this work we vary the distance of the tweezers between $a=5 \mu \mathrm{m}$ and $a=40 \mu \mathrm{m}$ (for details of the setup see Ref. [32]). When the ground state atoms are excited to the Rydberg state, they receive a recoil kick $\mathbf{p}_{r}=\hbar \mathbf{k}_{U V}$, which together with the repulsive ponderomotive force due 
(a)

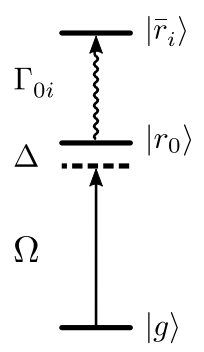

(b)

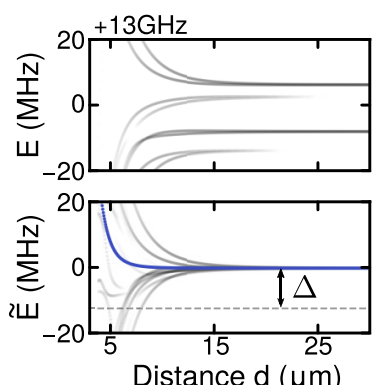

(c)

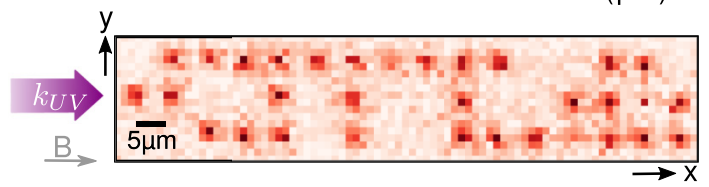

(d)

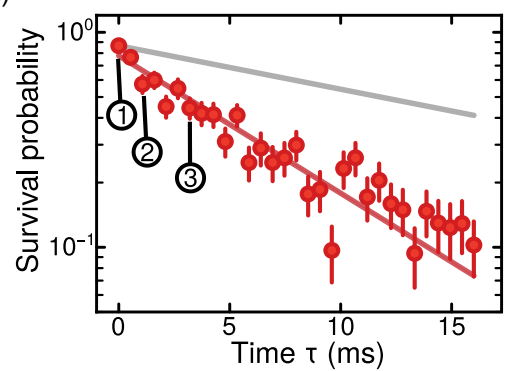

(e)

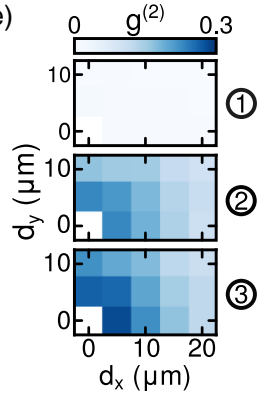

FIG. 1. (a) Simplified level scheme with the atomic ground state $|g\rangle$ and laser coupled Rydberg state $\left|r_{0}\right\rangle=\left|62 P_{1 / 2}, m_{J}=-1 / 2\right\rangle$ with Rabi frequency $\Omega$ and detuning $\Delta$. Blackbody radiation couples to nearby Rydberg states $\left|\bar{r}_{i}\right\rangle$ with rate $\Gamma_{0 i}$. (b) Van der Waals and dipolar pair-potentials. The dipole-dipole potentials between $\left|r_{0}\right\rangle$ and magnetic substates of the $13 \mathrm{GHz}$ higher lying, strongest coupled states $\left|61 D_{3 / 2}\right\rangle$ and $\left|61 D_{5 / 2}\right\rangle$ are shown in gray. The shading is proportional to the laser coupling rate for a magnetic field of $10 \mathrm{G}$, which mixes the finestructure states. In a blackbody event, the energy difference between $\left|r_{0}\right\rangle$ and $\left|\bar{r}_{i}\right\rangle$ is provided by the microwave photon, effectively collapsing all pair-potentials asymptotically (lower panel). The van der Waals potential between a pair of atoms in the state $\left|r_{0}\right\rangle$ is highlighted in blue in the lower panel, illustrating its much shorter range. (c) Single fluorescence image of ground state atoms in a $3 \times 16$ tweezer array with $5 \mu \mathrm{m}$ spacing. The magnetic field direction is indicated by the gray and the UV laser direction by the purple arrow. (d) Measurement of the trap lifetime in the $3 \times 16$ array at $\Delta=-2 \pi \times 4 \mathrm{MHz}$ and $\Omega=2 \pi \times 430 \mathrm{kHz}$. The dark-red line shows an exponential fit to the data revealing a trap lifetime of 6.8(4) ms, much shorter than the laser phase noise limited trap lifetime of 21.4(1.3) ms measured for individual atoms (gray line, see also Appendix Sec. 2. Error bars denote 1 s.e.m. (e) Two body correlator $g^{(2)}$ at different illumination times marked with the numbers in $\mathrm{d}$, showing the growth of correlations over time.

to the tweezers leads to efficient ejection out of the trap. We detect the lost atoms by comparison of two images, one before and one after the Rydberg laser illumination. The duration of the laser pulse is orders of magnitudes shorter than the vacuum limited trap lifetime of about $80 \mathrm{~s}$, such that lost atoms can be identified with Rydberg excitations.

In the limit of a low excitation fraction, any interaction induced line broadening can be understood in a two-atom picture, in which the presence of a contaminating atom leads to a distance-dependent level shift for nearby atoms. The

normally off resonant laser becomes resonant to the shifted atomic line if the interaction energy matches the detuning and the second atom is excited and subsequently lost from the trap. The line shifts may be rooted in van der Waals or dipolar interactions between two Rydberg atoms or in the electrostatic interaction between a Rydberg atom and an ion [33,34]. In all cases the process can lead to complex kinetically constrained dynamics [35-41], of which signatures have been observed by monitoring the bulk excitation dynamics of a Rydberg coupled gas [42-51]. The incoherent excitation is in contrast to a coherent two-photon excitation of the interacting pair, which becomes resonant at half the detuning. Facilitated excitation processes reduce the trap lifetime compared to the single atom expectation and imprint characteristic two-body correlations [see Figs. 1(d) and 1(e)] by which the underlying mechanism can be identified.

We measure the range of the induced two-point correlations on lost atoms $g^{(2)}(\mathbf{d})=\left\langle\left(n_{\mathbf{r}}-\left\langle n_{\mathbf{r}}\right\rangle\right)\left(n_{\mathbf{r}+\mathbf{d}}-\left\langle n_{\mathbf{r}+\mathbf{d}}\right\rangle\right)\right\rangle$ versus detuning from the atomic resonance. Here, $\mathbf{d}=\left(d_{x}, d_{y}\right)$ is the distance vector connecting the two tweezer positions, $n_{\mathbf{r}}= \pm 1$ encodes the occupation of the tweezer at position $\mathbf{r}$ and the averaging is over experimental runs and positions. We adjusted the Rabi frequency according to $\Omega / \Delta=\Omega_{m} / \Delta_{m}$ with maximum Rabi frequency $\Omega_{m}=2 \pi \times 0.4 \mathrm{MHz}$ at maximum detuning of $\Delta_{m}=-2 \pi \times 8 \mathrm{MHz}$, to limit the Rydberg state population $p_{0}=\alpha(\Delta) \Omega^{2} / 4 \Delta^{2}$, where $\alpha(\Delta)$ accounts for excess phase noise of the laser (see Appendix Sec. 2). We confirmed that the observed length scales are constant when decreasing $\Omega$ further. While the amplitude of $g^{(2)}$ is strongly dependent on the illumination time $\tau$, we found its spatial dependence to be insensitive to it. In order to assure comparability of the correlation amplitudes between different settings, we chose $\tau$ such that $60 \%$ of the initially loaded atoms remained in the array.

For correlations caused by blackbody-radiation-induced state contamination, the length scale of the correlations $g^{(2)}(d)$ is expected to be set by the pairwise dipolar interaction potentials. To extract a typical correlation length scale $d_{c}(\Delta)$ we fit the data exponentially with $g^{(2)}(d, \Delta) \propto \exp \left(-d / d_{c}(\Delta)\right)$. This empirical fit matches the data reasonably well (cf. inset of Fig. 2). The dipolar interaction potentials are approximately symmetric around the single atom resonance. In Fig. 2 we show that $d_{c}(\Delta)$ reproduces this approximate symmetry and we show that $d_{c}(\Delta)$ approximately matches the range of the dipolar pair potentials with a trend to a slight overestimation, which can be attributed to atomic motion as discussed below. To illustrate this, we plot the relevant dipolar potentials of pair eigenstates $\left\{\left|\Psi_{2}\right\rangle\right\}$ asymptotically correlating to $\left|r_{0}, \bar{r}_{i}\right\rangle$, with $\left|\bar{r}_{i}\right\rangle$, the $i$ th state of opposite parity populated by blackbody radiation with rate $\Gamma_{0 i}$. The energy difference $\hbar \Delta_{0 i}$ is provided by the blackbody photon and, hence, we plot all pair states asymptotically at the same energy as $\left|r_{0}, r_{0}\right\rangle$. The pair potentials are shaded according to the relative predicted facilitation strength. This is defined as the product $\left|\left\langle\Psi_{2} \mid r_{0}, \bar{r}_{i}\right\rangle\right|^{2} \Gamma_{0 i} / \Gamma_{0 k}^{\max }$ of the overlap of the pair state $\left|\Psi_{2}\right\rangle$ with $\left|r_{0}, \bar{r}_{i}\right\rangle$ and a normalized blackbody coupling rate $\Gamma_{0 i} / \Gamma_{0 k}^{\max }$. The normalization is with respect to the strongest coupled state with rate $\Gamma_{0 k}^{\max }$. For the calculations the pair interaction [52] and ARC [53] software packages have been used. 


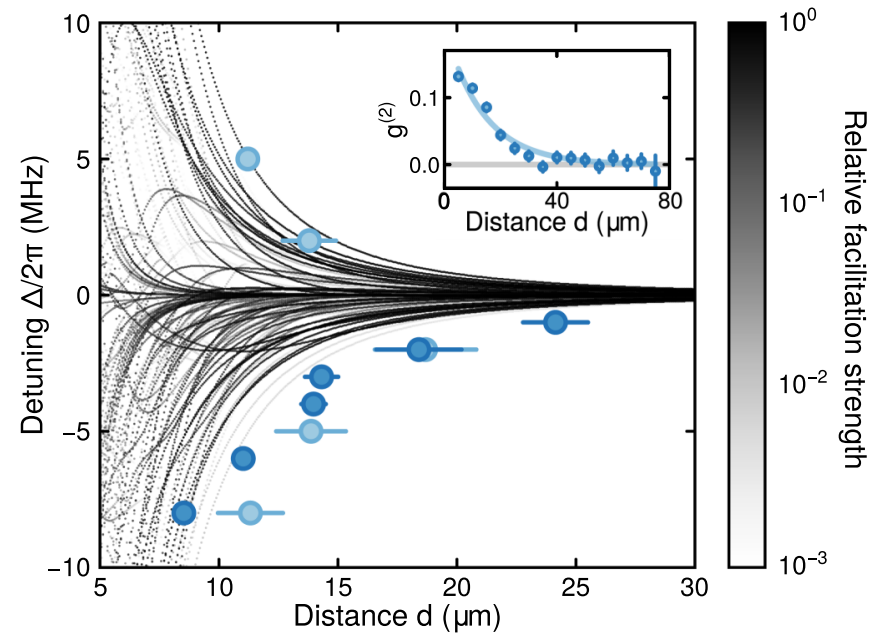

FIG. 2. Dipolar facilitation range. The figure shows the typical correlation distance $d_{c}(\Delta)$ extracted from an exponential fit to $g^{(2)}(d, \Delta)$ for a single line of atoms (light blue) and a $3 \times 16$ array (dark blue), both with spacing $a=5 \mu \mathrm{m}$. Gray shaded lines are the asymptotically collapsed dipolar pair-potentials with the shading proportional to the expected logarithmic facilitation strength. The inset shows an example of the detected two-body correlations with the exponential fit $g^{(2)}(d, \Delta) \propto \exp \left(-d / d_{c}(\Delta)\right)$ to extract $d_{c}(\Delta)$ for $\Delta=-2 \pi \times 5 \mathrm{MHz}$. Error bars denote 1 s.e.m. in the inset and the fit errors in the main panel.

While our analysis so far confirms that the length scale of the observed correlations is set by the dipolar pair potentials, our experiment fails to reproduce the short distance behavior expected in a picture of fixed atomic positions (see Appendix Sec. 4). This discrepancy is resolved when considering moving Rydberg atoms with trajectories determined by the interplay of temperature, atomic recoil, and the tweezer's ponderomotive potential. The atomic recoil velocity $v_{r}=\hbar k_{U V} / m=36 \mu \mathrm{m} / \mathrm{ms}$ for potassium atoms of mass $m$, due to scattering of photons from the UV laser, is comparable to the typical velocity gained from the ponderomotive potential $v_{U}=\sqrt{(2 \hbar U / m)}=40 \mu \mathrm{m} / \mathrm{ms}$, while the thermal velocity of $v_{T}=\sqrt{k_{B} T / m}=6.5 \mu \mathrm{m} / \mathrm{ms}$ for $T=200 \mathrm{nK}$ is much smaller. This results in a directed motion of the atoms exited to Rydberg states [Fig. 3(b)]. The decay to low lying states takes several hundred microseconds, in which the Rydberg atoms move by tens of micrometers, invalidating a static picture (see Appendix Sec. 3). The impact of this motion can be seen most directly when analyzing the trap lifetime locally [Fig. 3(d)]. Atoms in the first column of the array (counted with respect to the UV propagation direction) stay almost twice as long in the trap than atoms in the last column of the array. We attribute this to a lower effective facilitation rate as no facilitating atoms can approach from one direction. We confirmed that the signal is absent without UV illumination. To probe for the effect of this motion on the nearest-neighbor correlations we prepare arrays of different spacing $a$ and compare the strength of $g_{n n}^{(2)}(a)$ for two different detunings $\Delta=-2 \pi \times 2 \mathrm{MHz}$ and $\Delta=-2 \pi \times 5 \mathrm{MHz}$ [see Fig. 3(e)]. For those detunings, the dipolar potential range differs by almost a factor of two, but the observed distance dependence of $g_{n n}^{(2)}(a)$ is indistinguishable. This demonstrates (a)
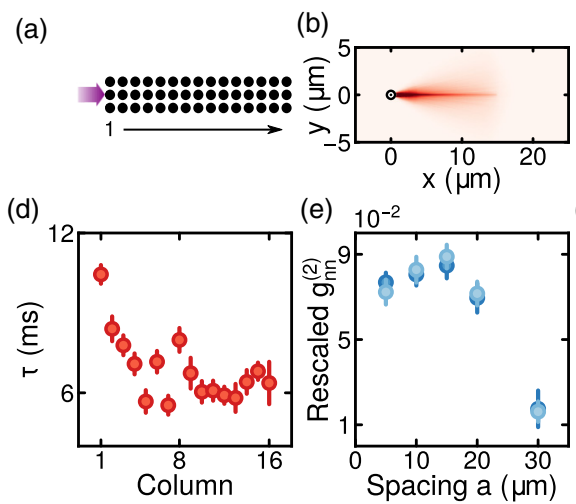

(e) $10^{-2}$
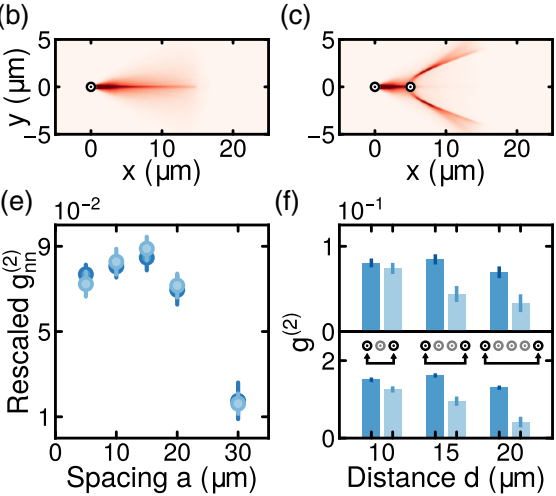

(f) $10^{-1}$

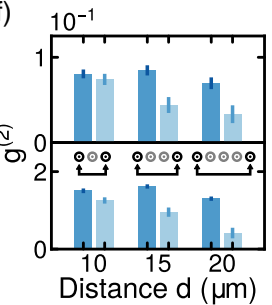

FIG. 3. The role of motion in the facilitation process. (a) Illustration of the geometry for the $3 \times 16$ array including the column numbering convention relative to the UV direction (purple arrow). (b) Ensemble of trajectories obtained from classical Monte Carlo simulations for the motion of the Rydberg atom. Shown are all trajectories in a window $\pm 3 \mu \mathrm{m}$ around the atomic plane. More trajectories crossing a point in space are indicated by lower lightness and the start position of the atoms is marked by the circular symbol. The apparent lines in the plot are artefacts of the simulation. (c) Same as in $\mathrm{b}$, but for an additional ponderomotive potential barrier present as indicated by the circular symbol. (d) Column-resolved trap lifetime measurement in a $5 \mu \mathrm{m}$ spaced $3 \times 16$ array for $\Omega=2 \pi \times 440 \mathrm{kHz}$ and $\Delta=-2 \pi \times 4 \mathrm{MHz}$. (e) Next-neighbor correlation $g_{n n}^{(2)}$ in a $1 \mathrm{D}$ chain of atoms versus the array spacing $a$ for detuning $\Delta=-2 \pi \times$ $5 \mathrm{MHz}$ (light blue) and $\Delta=-2 \pi \times 2 \mathrm{MHz}$ (dark blue). The data is scaled globally to the same average amplitude. (f) Histograms for $\Delta=-2 \pi \times 2 \mathrm{MHz}$ (top) and $\Delta=-2 \pi \times 5 \mathrm{MHz}$ (bottom) showing the effect of ponderomotive barriers in between two tweezers (see illustration). The dark (light) blue bars correspond to the case of no (at least one) ponderomotive barrier in between the tweezers at the respective distance. The correlation is calculated on a subset of the data, in which the tweezers in between (aka the ponderomotive barriers) where empty. Error bars denote 1 s.e.m.

that the sampling of all positions in flight is hiding the dependence of the dipolar pair potentials entirely, at least in the case of no other tweezers in the path of the atoms. The correlations feature a plateau for short distances, which we attribute to the typical flight distance of the Rydberg atoms within their electronic lifetime.

This result seems to be in contradiction to our observations reported in Fig. 2, which is resolved when taking into account the presence of other tweezers in the system. The recoil energy is about $3 \mu \mathrm{K}$, comparable to the ponderomotive potential height of $3.7 \mu \mathrm{K}$ of the individual tweezers, resulting in a "shielding" effect for the next-nearest neighbors. To support this interpretation we performed classical simulations, which confirm this effect [see Figs. 3(b) and 3(c)]. In Fig. 3(f) we show the strength of two-point correlations $g^{(2)}(d)$ for three distances $d$. For each distance, we compare the correlation amplitude of nearest-neighbor setting (zero potential barriers in between) to a setting of one or more potential barriers in between. In all cases, the setting without barriers in between shows strong and almost distance independent correlations. In contrast, when at least one potential barrier is present, the correlation amplitude decreases with distance and the effect is stronger for the larger detuning, for which the dipolar range 
(a)

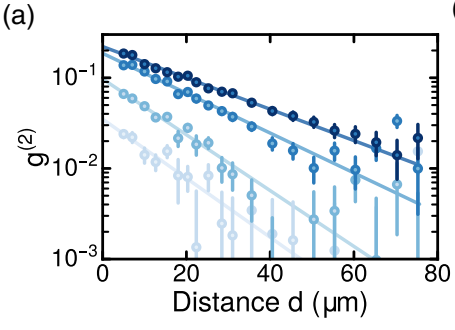

(b)

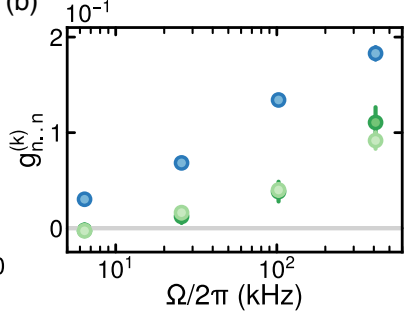

FIG. 4. Avalanche facilitation. (a). Two-point correlations $g^{(2)}$ vs distance for different coupling strength in a $5 \mu \mathrm{m}$-spaced $3 \times 16$ array. The Rabi frequency increases from light to dark blue as $\Omega=$ $2 \pi \times(6,25,102,410) \mathrm{kHz}$ and the pulse time was adjusted to fix the fraction of lost atoms to about 15\%. Exponential fits are shown as solid lines. (b). Multipoint correlations vs coupling strength. The shortest distance connected multi point correlations $g_{n . . n}^{(k)}$ in $x$ direction are shown for $k=2$ (blue), $k=3$ (light green) and $k=4$ (dark green). Error bars, where larger than the point size, denote 1 s.e.m.

is smaller. Note that in a static picture no dependence on the presence of empty traps in between the two positions is expected.

So far we have focused on the low excitation fraction regime, in which avalanche facilitation is small. The avalanche effect arises, when an already facilitated atom is transferred to a state $\left|\bar{r}_{i}\right\rangle$ by blackbody radiation and itself facilitates the excitation of further atoms. To test for the avalanche mechanism we measure the two-point correlations in the $3 \times 16$ geometry with $a=5 \mu \mathrm{m}$ for increasing Rabi frequency while fixing the total fraction of lost atoms. Figure 4(a) shows that both range and amplitude of two-point correlations increase with higher Rabi frequency. For the strongest driving, the amplitude of correlation between the two ends of the array (almost $80 \mu \mathrm{m}$ apart) is of comparable strength to the nearest-neighbor correlation for the weakest drive. The local measurements reveal that the strong increase of the $g^{(2)}(d)$ signal is accompanied by an emergence and subsequent increase of higher order correlations. In Fig. 4(b) we show the connected $k$-point correlator at shortest possible distance ( $k$ subsequent tweezers along the UV beam) $g_{n . . n}^{(k)}=$ $\left\langle\prod_{j=0}^{k}\left(n_{x+j a}-\left\langle n_{x+j a}\right\rangle\right)\right\rangle$. Remarkably, all higher order correlators increase simultaneously underlining the avalanche character of the process. This is further supported by a strong broadening of the distribution of lost atoms (see Appendix Sec. 5), which is a precursor of the observed bimodality in higher density settings $[28,54]$.

In this work we microscopically explored correlations emerging in atomic samples near resonantly coupled to Rydberg states. We identified dipolar interactions due to blackbody-radiation-induced state contamination as the underlying process. Furthermore, we have shown that the recoil triggered, directed motion of the Rydberg atoms is governing the facilitation process at nearest-neighbor distance. By increasing the driving strength, our observations seamlessly connect to previous studies, which concentrated on the avalanche regime in bulk systems [9-14]. The possibility to control the Rydberg motion by repulsive trapping potentials suggests the possibility of circumventing catastrophic avalanche dephasing in one- or two-dimensional Rydberg- dressed systems: With realistic experimental parameters a light-sheet potential at wavelengths chosen to trap the ground state but to repel the contaminant atoms can be implemented. This method works also in combination with tailored trapping wavelengths, which allow one to trap the ground state and only one particular Rydberg state [55,56]. It is compatible with future two-dimensional Rydberg quantum processors and simulators and, in particular, it paves the way to utilize Rydberg dressing for the design of atomic Hamiltonians for the study of various quantum spin models [21-23] or to generate useful states for quantum metrology [24-26].

\section{ACKNOWLEDGMENTS}

We acknowledge discussions with S. Hollerith and I. Lesanovsky. This project has received funding from the $\mathrm{Eu}-$ ropean Research Council (ERC) under Grant agreement No. 678580 (RyD-QMB) and the European Union's Horizon 2020 research and innovation program under Grant agreement No. 817482 (PASQuanS). We also acknowledge funding from Deutsche Forschungsgemeinschaft within SPP 1929 (GiRyd) and via a Heisenberg professorship to C.G., the MPG and support from the Alfried Krupp von Bohlen und Halbach foundation.

\section{APPENDIX: SUPPORTING DATA AND EXPERIMENTAL DETAILS}

\section{Experimental setting}

We load the ${ }^{39} \mathrm{~K}$ atoms from an optical molasses into the tweezer array and subsequently cool them near the motional ground state using Raman sideband cooling. We then ramp the optical tweezers down to $0.5 \%$ of their initial power (corresponding to a trap depth of $3.7 \mu \mathrm{K}$ ). This reduces the inhomogeneities between different tweezers to less than $50 \mathrm{kHz}$. Simultaneously, the atoms are adiabatically "cooled" to $200 \mathrm{nK}$, reducing the Doppler broadening to $2 \pi \times 50 \mathrm{kHz}$. This preparation of the atomic sample in the tweezer array is further detailed in Ref. [32].

\section{Single-atom trap lifetime and laser phase noise}

Spectral components of the phase noise of the Rydberg excitation laser, which match the detuning from resonance, increase the Rydberg population. The phase noise contribution often dominates the population, in particular, near the resonance. The impact of the phase noise alone can be conveniently revealed by measuring the single-atom trap lifetime, because any Rydberg excitation is efficiently ejected from the tweezer. In the main text we characterize this phase noise by an enhancement $\alpha(\Delta)$ of the Rydberg population w.r.t. the noise free value. The enhancement factor $\alpha(\Delta)=\tau_{i d} / \tau$ follows from the ratio of the measured trap lifetime $\tau$ and the ideal trap lifetime $\tau_{i d}=\tau_{r} \times 4 \Delta^{2} / \Omega^{2}$ for a noise-free laser. The latter is only limited by the electronic lifetime $\tau_{r}$ of the Rydberg state. Here we assumed $\Delta \gg \Omega$. In the tweezer array, isolated atoms can be realized by placing them far away from each other. We use distances of $30 \mu \mathrm{m}$ and $40 \mu \mathrm{m}$ and confirm that interactions can be neglected in this setting by checking for the absence of correlations in the losses. 
(a)

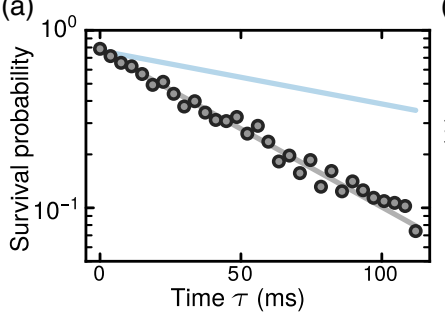

(b)

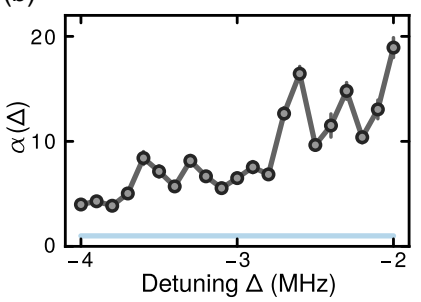

FIG. 5. Measurement of the laser noise reduced single atom lifetime. (a) Lifetime measurement (gray points) for single atoms with $30 \mu \mathrm{m}$ spacing for $\Omega=2 \pi \times 0.27 \mathrm{MHz}$ and $\Delta=-2 \pi \times 4 \mathrm{MHz}$. The gray line is an exponential fit to the data, the blue line is the expected decay for a noise-free laser. (b) Reduction $\alpha(\Delta)$ of the single atom lifetime as a function of the detuning. The blue line marks the ideal value $\alpha(\Delta)=1$

Figure 5 shows the result of a measurement of the trap lifetime for a Rabi frequency of $\Omega=2 \pi \times 266 \mathrm{kHz}$ and a detuning of $\Delta=-2 \pi \times 4 \mathrm{MHz}$. From the exponential fit we extract a lifetime of $\tau=49.3 \mathrm{~ms} \pm 1.1 \mathrm{~ms}$, resulting in a factor $\alpha=2.9$. The single-atom prediction shown in Fig. 1(d) of the main text includes this factor. In Fig. 5 we show $\alpha(\Delta)$, summarizing the results of all our noise characterization measurements.

\section{Electronic decay of the Rydberg state}

Figure 6 shows the decay rates of the chosen Rydberg state $62 P_{1 / 2}$ into all states of different principal quantum number for $0 \mathrm{~K}$ and for $300 \mathrm{~K}$. These numbers have been calculated with the ARC software package [53]. At $300 \mathrm{~K}$ the majority of decays are to nearby Rydberg states. When defining $n=30$ as a boundary between low and high lying states, of which the

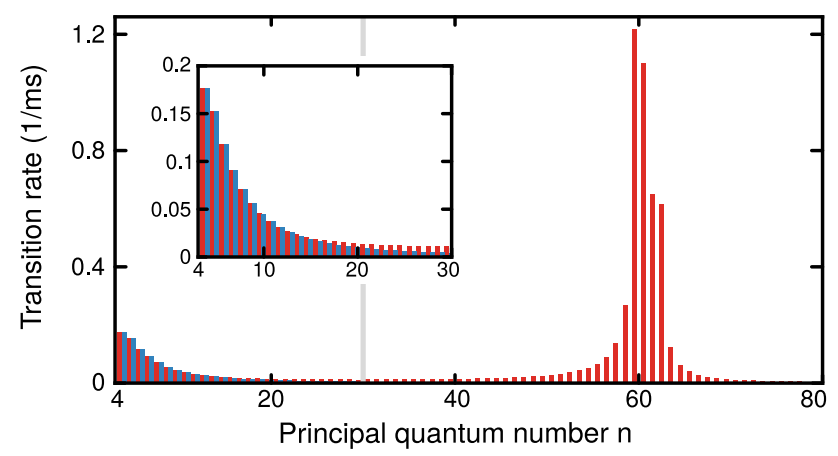

FIG. 6. Principal quantum number resolved decay rates of the $62 P_{1 / 2}$ state. The red histogram shows the decay rates to all final states taking into account the blackbody radiation background for a temperature of $300 \mathrm{~K}$. Comparison with the zero temperature histogram (blue) highlights the blackbody radiation triggered state changes (see also the inset). These are concentrated around the initial state, where dipole matrix elements are strongest. The gray line marks $n=30$, which we take as a boundary to estimate the total decay rate to facilitating and nonfacilitating states (see text). Blackbody radiation strongly increases the overall cumulative rate of decay from the initial state. (a)

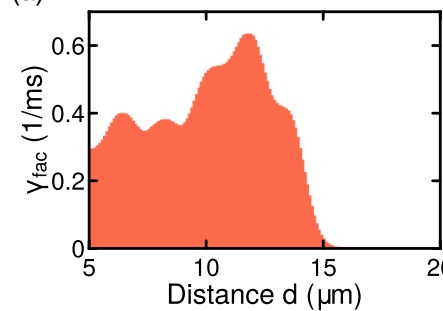

(b)

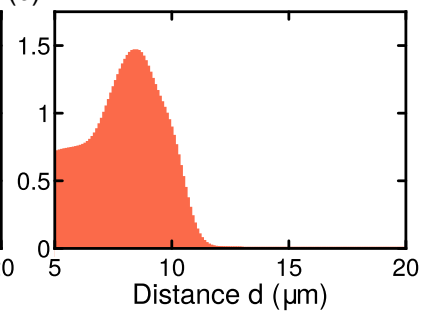

FIG. 7. Predicted facilitation rate $\gamma_{\mathrm{fac}}$ assuming fixed positions for the Rydberg atoms for $\Delta=-2 \pi \times 2 \mathrm{MHz}$ in (a) and for $\Delta=$ $-2 \pi \times 5 \mathrm{MHz}$ in (b). The spatial structure is determined by the crossing of dipolar pair potentials and the convolution with a gaussian function of standard deviation $\sigma=0.58 \mu \mathrm{m}$.

latter emerge only due to blackbody radiation, the ratio of the decay rates is $\sum_{n<30} \Gamma_{0, n} / \sum_{n \geqslant 30} \Gamma_{0, n}=\frac{2 \pi \times 0.16 \mathrm{kHz}}{2 \pi \times 0.8 \mathrm{kHz}} \approx \frac{1}{5}$.

Atoms, which make a blackbody radiation induced transition from the $62 P_{1 / 2}$ to a state of $s-$ or $d$-orbital symmetry interact via dipolar interactions with atoms in the $62 P_{1 / 2}$ state. This shifts the transition frequency and is the mechanism behind the facilitated excitation we observe. For the effects of the moving Rydberg atoms and for the avalanche processes at higher driving strength, the time the atoms stay in any Rydberg state is a fundamentally important parameter. This time can be approximated by the zero temperature lifetime, where all decays are to the low-lying states. In the vicinity of $n=60$, the $0 \mathrm{~K}$-lifetime is about $250 \mu \mathrm{s}$ for $s$ states, $800 \mu \mathrm{s}$ for $p$ states and $500 \mu \mathrm{s}$ for $d$ states. Including blackbody radiation, none of the states live for more than about $150 \mu \mathrm{s}$. When assuming a lifetime of $150 \mu$ s to roughly estimate the time in which facilitation can take place, the atoms move about $13 \mu \mathrm{m}$. Note that this is a crude simplification since the atoms may change their Rydberg state several times before decaying to the ground state.

\section{Dipolar facilitation for fixed atomic positions}

The resonant rate of dipolar facilitation $\gamma_{i}$ due to the $i$ th pair potential can be readily calculated in the low excitation fraction regime. It follows from $\gamma_{i}=\Gamma_{\text {eff }} p_{0} \Omega^{2} \tau_{r}^{2}$ with an effective rate $\Gamma_{\text {eff }}=\left|\left\langle\Psi_{2} \mid r_{0}, \bar{r}_{i}\right\rangle\right|^{2} \Gamma_{0, i}$, taking into account the pair-state
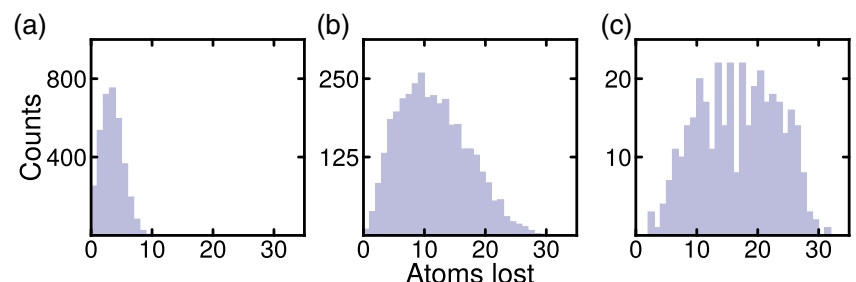

FIG. 8. Distribution of lost atoms for strong Rydberg driving at $\Delta=-2 \pi \times 3 \mathrm{MHz}$ and $\Omega=2 \pi \times 420 \mathrm{kHz}$. (a) Without UV exposure, where the loss is due to the imaging process. (b). Exposure time fixed such that $40 \%$ of the atoms are lost. (c) Same settings as for (b), but with postselection of the data to more than 26 atoms initially loaded. 
overlap $\left|\left\langle\Psi_{2} \mid r_{0}, \bar{r}_{i}\right\rangle\right|^{2}$ and the blackbody coupling rate $\Gamma_{0, i}$. The probability for the atom to be in state $\left|r_{0}\right\rangle$ is given by $p_{0}=\alpha(\Delta) \Omega^{2} / 4 \Delta^{2}$ and includes the laser phase noise factor $\alpha(\Delta)$. The electronic lifetime of the Rydberg state is $\tau_{r}$.

The expected facilitation rate $\gamma_{\text {fac }}$ shown in Fig. 7 for two detunings takes the rates $\gamma_{i}$ of all pair potentials into account, which become resonant at a certain distance. Additionally, it includes a convolution with a Gaussian of standard deviation $\sigma=0.58 \mu \mathrm{m}$ to include the thermal extent of the spatial wave function in the individual tweezers. The expected spatial dependence is clearly nonexponential in contradiction to our measurements. We interpret this as a further indication for the changing positions of the Rydberg atoms.

\section{Atom loss distributions at different Rabi frequency and density}

Previous experiments have reported a bimodality in the distribution of lost atoms and interpreted this as a signature of avalanche facilitation triggered by blackbody induced state contamination [28,54]. In Fig. 8 we show the distribution of lost atoms for different settings. It increases strongly in width with increasing Rabi frequency and even more when postselecting the high Rabi frequency data to the high density sector of more than 26 atoms (54\% of the tweezers) loaded. This matches the observation in previous experiments, in which the atomic density was even higher and the avalanche regime was fully realized.
[1] M. Saffman, T. G. Walker, and K. Mølmer, Quantum information with Rydberg atoms, Rev. Mod. Phys. 82, 2313 (2010).

[2] A. Browaeys and T. Lahaye, Many-body physics with individually controlled Rydberg atoms, Nat. Phys. 16, 132 (2020).

[3] T. M. Graham, M. Kwon, B. Grinkemeyer, Z. Marra, X. Jiang, M. T. Lichtman, Y. Sun, M. Ebert, and M. Saffman, RydbergMediated Entanglement in a Two-Dimensional Neutral Atom Qubit Array, Phys. Rev. Lett. 123, 230501 (2019).

[4] H. Levine, A. Keesling, G. Semeghini, A. Omran, T. T. Wang, S. Ebadi, H. Bernien, M. Greiner, V. Vuletić, H. Pichler, and M. D. Lukin, Parallel Implementation of High-Fidelity Multiqubit Gates with Neutral Atoms, Phys. Rev. Lett. 123, 170503 (2019).

[5] I. S. Madjarov, J. P. Covey, A. L. Shaw, J. Choi, A. Kale, A. Cooper, H. Pichler, V. Schkolnik, J. R. Williams, and M. Endres, High-fidelity entanglement and detection of alkalineearth Rydberg atoms, Nat. Phys. 16, 857 (2020).

[6] S. Ebadi, T. T. Wang, H. Levine, A. Keesling, G. Semeghini, A. Omran, D. Bluvstein, R. Samajdar, H. Pichler, W. W. Ho, S. Choi, S. Sachdev, M. Greiner, V. Vuletić, and M. D. Lukin, Quantum phases of matter on a 256-atom programmable quantum simulator, Nature (London) 595, 227 (2021).

[7] P. Scholl, M. Schuler, H. J. Williams, A. A. Eberharter, D. Barredo, K.-N. Schymik, V. Lienhard, L.-P. Henry, T. C. Lang, T. Lahaye, A. M. Läuchli, and A. Browaeys, Quantum simulation of 2D antiferromagnets with hundreds of Rydberg atoms, Nature (London) 595, 233 (2021).

[8] I. I. Beterov, I. I. Ryabtsev, D. B. Tretyakov, and V. M. Entin, Quasiclassical calculations of blackbody-radiation-induced depopulation rates and effective lifetimes of Rydberg $n S, n P$, and $n D$ alkali-metal atoms with $n \leqslant 80$, Phys. Rev. A 79, 052504 (2009).

[9] E. A. Goldschmidt, T. Boulier, R. C. Brown, S. B. Koller, J. T. Young, A. V. Gorshkov, S. L. Rolston, and J. V. Porto, Anomalous Broadening in Driven Dissipative Rydberg Systems, Phys. Rev. Lett. 116, 113001 (2016).

[10] B. J. DeSalvo, J. A. Aman, C. Gaul, T. Pohl, S. Yoshida, J. Burgdörfer, K. R. A. Hazzard, F. B. Dunning, and T. C. Killian, Rydberg-blockade effects in Autler-Townes spectra of ultracold strontium, Phys. Rev. A 93, 022709 (2016).

[11] J. A. Aman, B. J. DeSalvo, F. B. Dunning, T. C. Killian, S. Yoshida, and J. Burgdörfer, Trap losses induced by near- resonant Rydberg dressing of cold atomic gases, Phys. Rev. A 93, 043425 (2016).

[12] C. Gaul, B. J. DeSalvo, J. A. Aman, F. B. Dunning, T. C. Killian, and T. Pohl, Resonant Rydberg Dressing of Alkaline-Earth Atoms via Electromagnetically Induced Transparency, Phys. Rev. Lett. 116, 243001 (2016).

[13] T. Boulier, E. Magnan, C. Bracamontes, J. Maslek, E. A. Goldschmidt, J. T. Young, A. V. Gorshkov, S. L. Rolston, and J. V. Porto, Spontaneous avalanche dephasing in large Rydberg ensembles, Phys. Rev. A 96, 053409 (2017).

[14] J. de Hond, N. Cisternas, R. J. C. Spreeuw, H. B. van Linden van den Heuvell, and N. J. v. Druten, Interplay between van der Waals and dipole-dipole interactions among Rydberg atoms, J. Phys. B 53, 084007 (2020).

[15] J. T. Young, T. Boulier, E. Magnan, E. A. Goldschmidt, R. M. Wilson, S. L. Rolston, J. V. Porto, and A. V. Gorshkov, Dissipation-induced dipole blockade and antiblockade in driven Rydberg systems, Phys. Rev. A 97, 023424 (2018).

[16] L. Santos, G. V. Shlyapnikov, P. Zoller, and M. Lewenstein, Bose-Einstein Condensation in Trapped Dipolar Gases, Phys. Rev. Lett. 85, 1791 (2000).

[17] I. Bouchoule and K. Mølmer, Spin squeezing of atoms by the dipole interaction in virtually excited Rydberg states, Phys. Rev. A 65, 041803(R) (2002).

[18] N. Henkel, R. Nath, and T. Pohl, Three-Dimensional Roton Excitations and Supersolid Formation in Rydberg-Excited Bose-Einstein Condensates, Phys. Rev. Lett. 104, 195302 (2010).

[19] G. Pupillo, A. Micheli, M. Boninsegni, I. Lesanovsky, and P. Zoller, Strongly Correlated Gases of Rydberg-Dressed Atoms: Quantum and Classical Dynamics, Phys. Rev. Lett. 104, 223002 (2010).

[20] J. E. Johnson and S. L. Rolston, Interactions between Rydbergdressed atoms, Phys. Rev. A 82, 033412 (2010).

[21] R. M. W. van Bijnen and T. Pohl, Quantum Magnetism and Topological Ordering via Rydberg Dressing near Förster Resonances, Phys. Rev. Lett. 114, 243002 (2015).

[22] A. W. Glaetzle, M. Dalmonte, R. Nath, C. Gross, I. Bloch, and P. Zoller, Designing Frustrated Quantum Magnets with LaserDressed Rydberg Atoms, Phys. Rev. Lett. 114, 173002 (2015).

[23] I.-D. Potirniche, A. C. Potter, M. Schleier-Smith, A. Vishwanath, and N. Y. Yao, Floquet Symmetry-Protected 
Topological Phases in Cold-Atom Systems, Phys. Rev. Lett. 119, 123601 (2017).

[24] L. I. R. Gil, R. Mukherjee, E. M. Bridge, M. P. A. Jones, and T. Pohl, Spin Squeezing in a Rydberg Lattice Clock, Phys. Rev. Lett. 112, 103601 (2014).

[25] R. Kaubruegger, P. Silvi, C. Kokail, R. van Bijnen, A. M. Rey, J. Ye, A. M. Kaufman, and P. Zoller, Variational Spin-Squeezing Algorithms on Programmable Quantum Sensors, Phys. Rev. Lett. 123, 260505 (2019).

[26] R. Kaubruegger, D. V. Vasilyev, M. Schulte, K. Hammerer, and P. Zoller, Quantum Variational Optimization of Ramsey Interferometry and Atomic Clocks, Phys. Rev. X 11, 041045 (2021).

[27] Y.-Y. Jau, A. M. Hankin, T. Keating, I. H. Deutsch, and G. W. Biedermann, Entangling atomic spins with a Rydberg-dressed spin-flip blockade, Nat. Phys. 12, 71 (2016).

[28] J. Zeiher, R. van Bijnen, P. Schauß, S. Hild, J.-y. Choi, T. Pohl, I. Bloch, and C. Gross, Many-body interferometry of a Rydbergdressed spin lattice, Nat. Phys. 12, 1095 (2016).

[29] J. Zeiher, J.-Y. Choi, A. Rubio-Abadal, T. Pohl, R. van Bijnen, I. Bloch, and C. Gross, Coherent Many-Body Spin Dynamics in a Long-Range Interacting Ising Chain, Phys. Rev. X 7, 041063 (2017).

[30] E. Guardado-Sanchez, B. M. Spar, P. Schauss, R. Belyansky, J. T. Young, P. Bienias, A. V. Gorshkov, T. Iadecola, and W. S. Bakr, Quench Dynamics of a Fermi Gas with Strong Nonlocal Interactions, Phys. Rev. X 11, 021036 (2021).

[31] V. Borish, O. Marković, J. A. Hines, S. V. Rajagopal, and M. Schleier-Smith, Transverse-Field Ising Dynamics in a Rydberg-Dressed Atomic Gas, Phys. Rev. Lett. 124, 063601 (2020).

[32] N. Lorenz, L. Festa, L.-M. Steinert, and C. Gross, Raman sideband cooling in optical tweezer arrays for Rydberg dressing, SciPost Phys. 10, 052 (2021).

[33] D. Weller, A. Urvoy, A. Rico, R. Löw, and H. Kübler, Chargeinduced optical bistability in thermal Rydberg vapor, Phys. Rev. A 94, 063820 (2016).

[34] A. D. Bounds, N. C. Jackson, R. K. Hanley, E. M. Bridge, P. Huillery, and M. P. A. Jones, Coulomb anti-blockade in a Rydberg gas, New J. Phys. 21, 053026 (2019).

[35] C. Ates, T. Pohl, T. Pattard, and J. M. Rost, Antiblockade in Rydberg Excitation of an Ultracold Lattice Gas, Phys. Rev. Lett. 98, 023002 (2007).

[36] M. Gärttner, K. P. Heeg, T. Gasenzer, and J. Evers, Dynamic formation of Rydberg aggregates at off-resonant excitation, Phys. Rev. A 88, 043410 (2013).

[37] I. Lesanovsky and J. P. Garrahan, Out-of-equilibrium structures in strongly interacting Rydberg gases with dissipation, Phys. Rev. A 90, 011603(R) (2014).

[38] M. Mattioli, A. W. Glätzle, and W. Lechner, From classical to quantum non-equilibrium dynamics of Rydberg excitations in optical lattices, New J. Phys. 17, 113039 (2015).

[39] M. Marcuzzi, M. Buchhold, S. Diehl, and I. Lesanovsky, Absorbing State Phase Transition with Competing Quantum and Classical Fluctuations, Phys. Rev. Lett. 116, 245701 (2016).

[40] F. Letscher, O. Thomas, T. Niederprüm, H. Ott, and M. Fleischhauer, Anomalous excitation facilitation in inhomogeneously broadened Rydberg gases, Phys. Rev. A 95, 023410 (2017).
[41] K. Klocke and M. Buchhold, Controlling excitation avalanches in driven Rydberg gases, Phys. Rev. A 99, 053616 (2019).

[42] C. Carr, R. Ritter, C. G. Wade, C. S. Adams, and K. J. Weatherill, Nonequilibrium Phase Transition in a Dilute Rydberg Ensemble, Phys. Rev. Lett. 111, 113901 (2013).

[43] H. Schempp, G. Günter, M. Robert-de Saint-Vincent, C. S. Hofmann, D. Breyel, A. Komnik, D. W. Schönleber, M. Gärttner, J. Evers, S. Whitlock, and M. Weidemüller, Full Counting Statistics of Laser Excited Rydberg Aggregates in a One-Dimensional Geometry, Phys. Rev. Lett. 112, 013002 (2014).

[44] N. Malossi, M. M. Valado, S. Scotto, P. Huillery, P. Pillet, D. Ciampini, E. Arimondo, and O. Morsch, Full Counting Statistics and Phase Diagram of a Dissipative Rydberg Gas, Phys. Rev. Lett. 113, 023006 (2014).

[45] A. Urvoy, F. Ripka, I. Lesanovsky, D. Booth, J. Shaffer, T. Pfau, and R. Löw, Strongly Correlated Growth of Rydberg Aggregates in a Vapor Cell, Phys. Rev. Lett. 114, 203002 (2015).

[46] M. M. Valado, C. Simonelli, M. D. Hoogerland, I. Lesanovsky, J. P. Garrahan, E. Arimondo, D. Ciampini, and O. Morsch, Experimental observation of controllable kinetic constraints in a cold atomic gas, Phys. Rev. A 93, 040701(R) (2016).

[47] N. R. de Melo, C. G. Wade, N. Šibalić, J. M. Kondo, C. S. Adams, and K. J. Weatherill, Intrinsic optical bistability in a strongly driven Rydberg ensemble, Phys. Rev. A 93, 063863 (2016)

[48] R. Gutiérrez, C. Simonelli, M. Archimi, F. Castellucci, E. Arimondo, D. Ciampini, M. Marcuzzi, I. Lesanovsky, and O. Morsch, Experimental signatures of an absorbing-state phase transition in an open driven many-body quantum system, Phys. Rev. A 96, 041602(R) (2017).

[49] F. Letscher, O. Thomas, T. Niederprüm, M. Fleischhauer, and H. Ott, Bistability Versus Metastability in Driven Dissipative Rydberg Gases, Phys. Rev. X 7, 021020 (2017).

[50] S. Helmrich, A. Arias, G. Lochead, T. M. Wintermantel, M. Buchhold, S. Diehl, and S. Whitlock, Signatures of self-organized criticality in an ultracold atomic gas, Nature (London) 577, 481 (2020).

[51] D.-S. Ding, H. Busche, B.-S. Shi, G.-C. Guo, and C. S. Adams, Phase Diagram and Self-Organizing Dynamics in a Thermal Ensemble of Strongly Interacting Rydberg Atoms, Phys. Rev. X 10, 021023 (2020).

[52] S. Weber, C. Tresp, H. Menke, A. Urvoy, O. Firstenberg, H. P. Büchler, and S. Hofferberth, Calculation of Rydberg interaction potentials, J. Phys. B 50, 133001 (2017).

[53] N. Šibalić, J. Pritchard, C. Adams, and K. Weatherill, ARC: An open-source library for calculating properties of alkali Rydberg atoms, Comput. Phys. Commun. 220, 319 (2017).

[54] N. Thaicharoen, S. A. Miller, and G. Raithel, Expansion behavior and pair correlations in continuously excited Rydberg systems, Phys. Rev. A 98, 023402 (2018).

[55] M. Saffman and T. G. Walker, Analysis of a quantum logic device based on dipole-dipole interactions of optically trapped Rydberg atoms, Phys. Rev. A 72, 022347 (2005).

[56] L. Li, Y. O. Dudin, and A. Kuzmich, Entanglement between light and an optical atomic excitation, Nature (London) 498, 466 (2013). 